\title{
Photo-Controlled Adhesives Based on Photoinduced Solid-to-Liquid Transition of an Azobenzene Compound
}

\author{
Bing Niu ${ }^{a, b}$ (D) \\ Mingsen Chen ${ }^{a, b, c, d}$ (iD \\ Xiaolei Zhao ${ }^{\text {a (iD) }}$ \\ Yuanli Liu*a (iD \\ Si Wu ${ }^{* a, b, c}$ (iD)

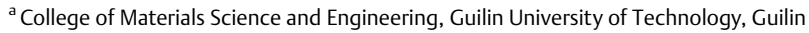 \\ 541000, China \\ ${ }^{\mathrm{b}}$ CAS Key Laboratory of Soft Matter Chemistry, Hefei National Laboratory for Physical \\ Sciences at the Microscale, Department of Polymer Science and Engineering, \\ University of Science and Technology of China, Hefei 260026, China \\ 'Max Planck Institute for Polymer Research, 55128 Mainz, Germany \\ department of Chemical Engineering, Tsinghua University, Beijing, China \\ lyuanli@glut.edu.cn; siwu@ustc.edu.cn
}

Received: 02.10.2020

Accepted after revision: 19.11.2020

DOI: 10.1055/s-0040-1721756; Art ID: om-20-0033oa

License terms: CC

(c) 2020. The Author(s). This is an open access article published by Thieme under the terms of the Creative Commons Attribution License, permitting unrestricted use, distribution, and reproduction so long as the original work is properly cited. (https:// creativecommons.org/licenses/by/4.0/)

Abstract The development of photo-controlled adhesives can overcome the problems associated with daily lives and industrial applications. Adhesion is a multidiscipline field of engineering, physics, and chemistry. The solid-to-liquid transformation of light-controlled adhesives can be used for direct bonding onto diverse surfaces. Here, a photoresponsive azobenzene compound is developed for photocontrolled adhesion. The azobenzene compound 4, 4'-hexyl diacrylate-3-methylazobenzene (M1) exhibits photoinduced solid-to-liquid transition due to trans-cis photoisomerization. The prepolymer coating based on the azobenzene compound $\mathbf{M} \mathbf{1}$ is prepared on an adhesive surface. After UV irradiation, the solid coating was quickly transformed into liquid for adhesion. This photo-controlled adhesive has strong adhesion to different surfaces.

Key words azobenzenes, photoinduced solid-to-liquid transition prepolymer coating, photo-controlled adhesives

\section{Introduction}

Many adhesives are liquids, ${ }^{1}$ including water-soluble adhesives, ${ }^{2}$ solvent-based adhesives, ${ }^{3}$ emulsion adhesives, ${ }^{4}$ and so on. For industrial applications, the glue coating process is often needed, which is time-consuming and causes bonding loss due to improper sizing. Therefore, there is an urgent need for more convenient adhesives in practical applications. The design of smart surfaces with switchable adhesive properties has remained a challenge in adhesion science and materials engineering. ${ }^{5}$ Lightcontrolled adhesives ${ }^{6}$ have some advantages, such as

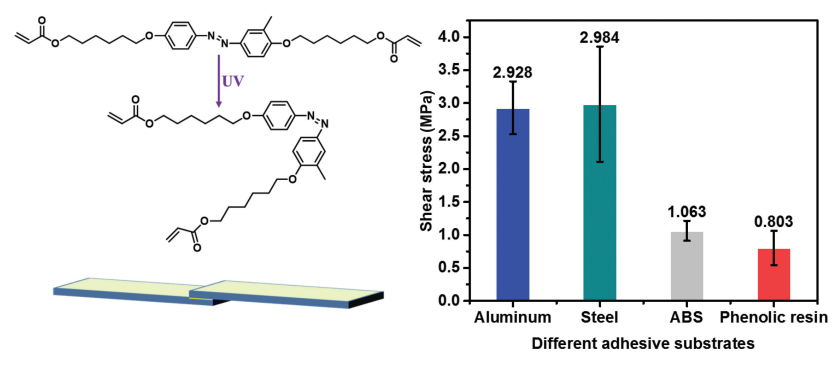

noncontact stimulus response, cleanliness, and convenience of light energy, which will be the trend for the development of adhesives. ${ }^{7}$

Azobenzene is a switchable compound with reversible cis-trans photoisomerization abilities. ${ }^{8}$ Photoresponsive azobenzene compounds have been used for applications in information storage, ${ }^{9}$ lithography, ${ }^{10}$ solar energy storage, ${ }^{11}$ actuators, ${ }^{12}$ and so on. At present, azobenzene molecules are widely studied. Some azobenzene compounds are solids in the trans-state with a melting point higher than room temperature $^{13,14}$; however, when irradiated with UV light, they can be transformed into liquids in the cis-state with a melting point lower than room temperature. Photoisomerization of some azobenzene small molecules can reversibly convert the compounds from solids to isotropic liquids. ${ }^{15}$ In this study, a photoinduced solid-liquid transition adhesive prepolymer coating was prepared based on the special properties of azobenzene compounds. After UV irradiation, the solid coating was quickly transformed into liquid for adhesion. This kind of photo-controlled adhesive coating brings great convenience for industrial and daily-life applications.

\section{Results and Discussion}

We prepared an azobenzene compound that is effective for photoinduced solid-liquid phase transition. The solid-toliquid phase transition is one of the most fundamental phenomena in materials science. It is a process in which the intermolecular forces, such as van der Waals, present in the solid state weaken at a certain temperature or pressure. However, little attention has been paid to the "photo-melting" phenomenon ${ }^{16}$ despite a rich functionality of these photo-melting compounds. In our study, we found that azobenzene compound M1 (synthesis and ${ }^{1} \mathrm{H}$ NMR spectra of the azobenzene compound M1; Supporting Information, Figures S1 and S2) can undergo a solid-to-liquid 


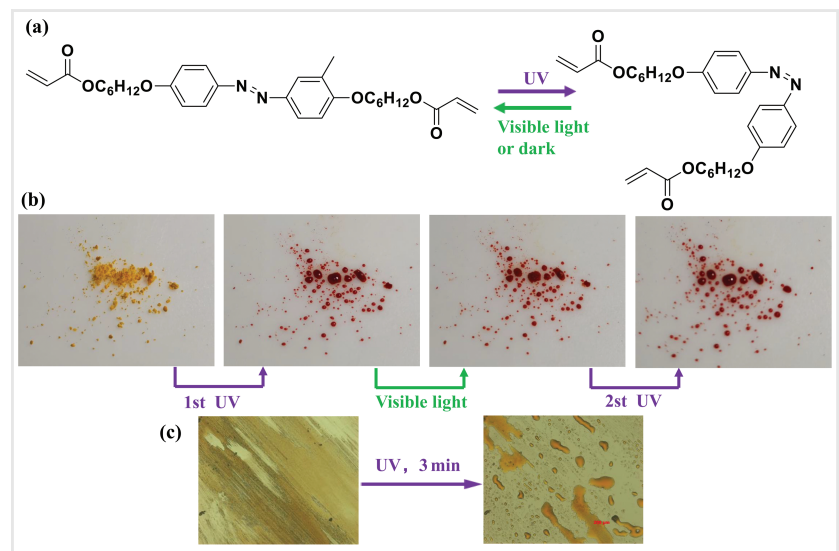

Figure 1 (a) Chemical structure and photoisomerization of azobenzene compound M1. (b) Photographs of photoinduced solid-to-liquid transition of azobenzene M1 (UV for $3 \mathrm{~min}$; visible light for $5 \mathrm{~min}$ ). (c) Optical microscopy images of photoinduced $\left(365 \mathrm{~nm}, 55.6 \mathrm{~mW} \mathrm{~cm}{ }^{-2}\right.$, $10 \mathrm{~min}$ ) solid-to-liquid transition of $\mathbf{M} 1$.

transition under UV light at room temperature (Figure 1). In Figure 1a, we selected a rod-shaped molecule with double bonds at the ends, and introduced a methyl group at the 3position. These asymmetric molecules have been carefully discussed in a study by Akiyama et al. ${ }^{17}$ The introduction of this asymmetric methyl can weaken the $\pi-\pi$ interaction in the molecule, which weakens the compactness of the molecular packing. ${ }^{18}$ The introduction of this asymmetric methyl also weakens the $\mathrm{H}$-aggregation in the molecules. ${ }^{19}$ The introduction of long alkyl chains at both ends increases the flexibility of azobenzene molecules, and makes azobenzene molecules more prone to photoisomerization. ${ }^{20}$ Under UV irradiation, the lattice structure of small molecules is broken, then isomerizes to a cis-structure and quickly changes to liquids. Under irradiation of visible light or keeping it in the dark, it can be restored to a trans-form, which is solid.

The trans-cis photoisomerization behavior of the azobenzene compound induces macroscopic motions and phase changes of materials. ${ }^{21}$ As shown in Figure $1 \mathrm{~b}$, the compound M1 begins to liquefy at $30 \mathrm{~s}$ under UV irradiation at an intensity of $55.6 \mathrm{~mW} \mathrm{~cm}^{-2}$, and can be completely transformed into liquid after $3 \mathrm{~min}$ of irradiation. Compound M1 returned to the solid state after $5 \mathrm{~min}$ of visible light irradiation. After irradiation with UV light again, compound M1 becomes liquid again, and it can also return to the solid state by spontaneous thermal relaxation in the dark. Compound M1 showed an interesting photoinduced solid-to-liquid transition. Under an optical microscope, M1 powders were illuminated with $365 \mathrm{~nm}$ UV light (Figure 1c). UV illumination changed the irregularly shaped $\mathbf{M 1}$ powders into drops, showing photo-liquefaction. This observation indicates that UV illumination induced liquidation of the compound.

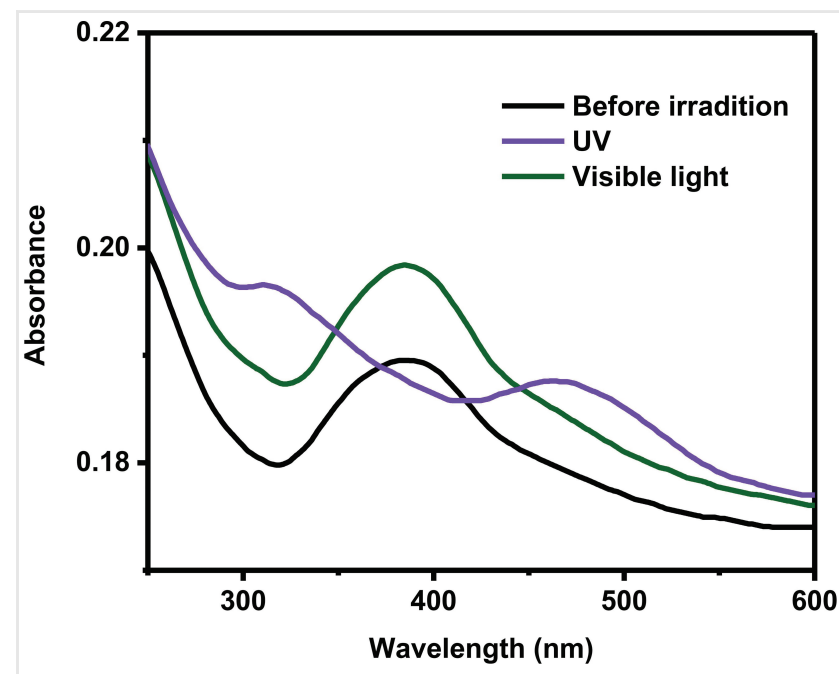

Figure 2 UV-vis absorption spectra of $\mathbf{M} \mathbf{1}$ before irradiation, after UV irradiation ( $365 \mathrm{~nm}, 43.6 \mathrm{~mW} \mathrm{~cm}{ }^{-2}, 3 \mathrm{~min}$ ), and subsequent visible light irradiation ( $530 \mathrm{~nm}, 13 \mathrm{~mW} \mathrm{~cm}{ }^{-2}, 3 \mathrm{~min}$ ).

UV-vis absorption spectroscopy showed that a thin film of M1 exhibits reversible cis-trans photoisomerization (Figure 2). The azobenzene groups in the as-prepared film were in the stable trans-state and exhibited a $\pi-\pi^{*}$ band at $385 \mathrm{~nm}$. Upon UV irradiation, the $\mathrm{n}-\pi^{*}$ band of the cis-isomer at $465 \mathrm{~nm}$ increased. Subsequently, the film was irradiated with visible light, which switched it back to the trans-state, ${ }^{22}$ resulting in the recovery of the absorption peak at $455 \mathrm{~nm}$. The sample can also be restored to the trans-structure by keeping in the dark at room temperature (Figure S3). The absorbance of M1 before irradiation was lower than that of M1 after irradiated with visible light because the stacking and orientation of the azobenzene groups changed. ${ }^{23}$ Despite the in-depth study of azobenzene adhesives in recent years, ${ }^{24-28}$ the bonding strength has not been greatly improved, which cannot meet the needs of industrial applications. The prepolymer coating we prepared includes both azobenzene as a light-responsive switch and a reinforced resin, which can be used to prepare a high-strength photo-controlled adhesive. The prepolymer coating was prepared on an aluminum plate by UV-induced solid-to-liquid phase transition of M1. The prepolymer coating includes the switchable molecule M1, reinforced resin polyurethane-acrylate (PUA), hydroxypropyl methacrylate (HPMA), accelerants 1-acetyl-2-phenylhydrazine (APH) and saccharin (SAC), anaerobic initiator cumene hydroperoxide (MSDS), and polymerization inhibitor hydroquinone (HQ). The mass ratio of each component is shown in Table 1. In the composition of the adhesive, PUA and HPMA provide adhesion through copolymerization, MSDS initiates polymerization, and azobenzene compound $\mathbf{M} \mathbf{1}$ acts as a light switch.

The prepared prepolymer coating is solid at room temperature and can be rapidly transformed into liquid 
Organic Materials

B. Niu et al.

Table 1 Mass ratio of azobenzene adhesives

\begin{tabular}{lllll}
\hline & 1 & 2 & 3 & 4 \\
\hline M1 (wt\%) & 96.5 & 86.5 & 76.5 & 46.5 \\
HPMA (wt\%) & 0 & 5 & 10 & 20 \\
PUA (wt\%) & 0 & 5 & 10 & 20 \\
APH (wt\%) & 0.4 & 0.4 & 0.4 & 0.4 \\
SAC (wt\%) & 0.6 & 0.6 & 0.6 & 0.6 \\
MSDS (wt\%) & 2 & 1 & 2 & 2 \\
HQ (wt\%) & 0.5 & 0.5 & 0.5 & 0.5 \\
\hline
\end{tabular}

by UV irradiation, which can be used for anaerobic polymerization and bonding with another substrate. In the process of polymerization and bonding, the lack of oxygen in the bonding interface leads to the production of free radicals of the anaerobic initiator ${ }^{29}$ MSDS, which leads to the anaerobic polymerization of the bonding system to form tough bonding. As shown in Figure 3a, the prepolymer coating can be liquefied in 3 min by irradiating it with UV light. In the whole process, azobenzene monomer M1 is used as a switch for optically controlled solid-to-liquid conversion. This photoinduced solid-to-liquid transition can also be clearly observed in the optical microscope photograph (Figure 3b). Finally, we tested the adhesion based on the prepolymer coating. The prepolymer coating was liquefied immediately after being illuminated by UV light. Another substrate was pressed onto the adhesive to create an anaerobic environment. In the absence of oxygen and the presence of APH and SAC, MSDS produces initial free radicals to initiate chain growth, which are then crosslinked and cured. The adhesion model is shown in Figure 4a. We used the tensile shear strength test model to measure

(a)
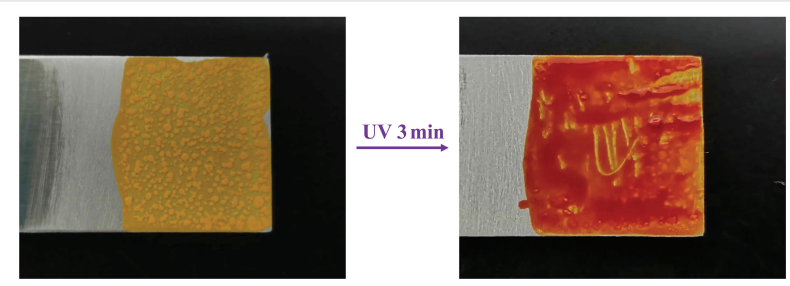

(b)
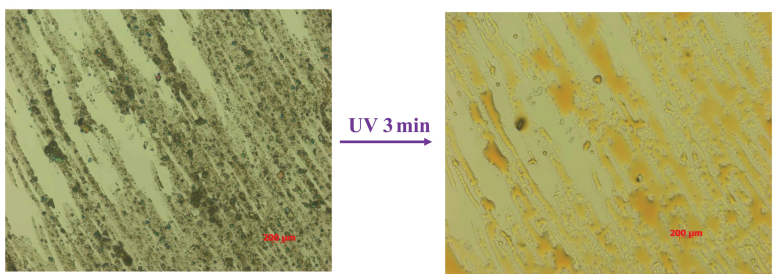

Figure 3 Photoinduced ( $365 \mathrm{~nm}, 38.9 \mathrm{~mW} \mathrm{~cm}{ }^{-2}, 3 \mathrm{~min}$ ) solid-toliquid transition of (a) adhesive coating under the macroscopic state and (b) under an optical microscope (56.5 wt\% azobenzene compound M1).

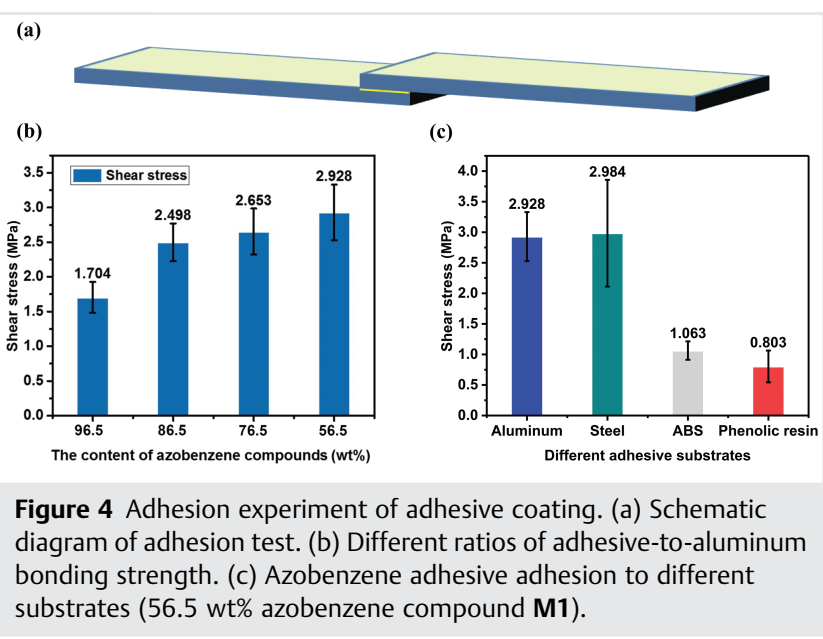

the adhesion (tensile shear strength using universal testing machine, Figure S4).

As shown in Figure $4 \mathrm{~b}$, we tested the shear strength of adhesive bonding aluminum plates with different contents of azobenzene compound M1, and then determined the optimal binder composition ratio. When the system contained only the azobenzene compound M1, the shear strength of the adhesive aluminum plate was $1.704 \mathrm{MPa}$. In the bonding system, M1 was used as the switch for the photo-controlled solid-liquid transition. The shear strength was mainly provided by the reinforced resin PUA and polymerized HPMA. With the decrease of M1 content and the increase of PUA and HPMA contents, the shear strengths increased. When the mass ratio of azobenzene monomer M1 was $56.5 \mathrm{wt} \%$ and the mass ratios of PUA and HPMA were $20 \%$, the shear strength of the aluminum plate reached $2.928 \mathrm{MPa}$. In order to prove the stability of the adhesive prepared by us in the daylight, we first stick aluminum plates with the photo-controlled adhesive ( $56.5 \mathrm{wt} \%$ M1), and then leave it in the daylight for 3 days to test the adhesion force. The corresponding tensile curve is shown in Figure S5, and the shear strength can reach $2.77 \mathrm{MPa}$. It is proved that the adhesive has good stability.

To demonstrate the universality of this light-controlled adhesive, the shear strength of the adhesive to the aluminum plate, steel plate, ABS resin, and phenolic resin was tested. Our photo-controlled adhesives can bond steel plates, ABS resin, and phenolic resin.

\section{Conclusions}

In conclusion, we prepared photo-controlled adhesives based on an azobenzene compound, M1. We prepared the adhesive substrate using a prepolymer coating. Based on the photoinduced solid-to-liquid transition of M1, the prepolymer coating can be liquefied immediately by UV light 
irradiation, and then adhered to the other substrate by anaerobic polymerization. In our work, azobenzene molecules as a kind of optical switch give the photo-controlled properties of the adhesive, and the bonding strength is mainly provided by reinforced resins and monomers. Our light-controlled adhesive can show powerful adhesion performance and excellent adhesion to different interfaces.

\section{Experimental Section}

\section{Reagents}

O-Cresol, 4-aminophenol, and 6-chloro-1-hexanol were purchased from Shanghai Adamas Reagent (Shanghai, China). Triethylamine, acryloyl chloride, and sodium nitrite were supplied by Adamas Reagent (Shanghai, China). Hydrochloric acid $(\mathrm{HCl})$, sodium hydroxide $(\mathrm{NaOH})$, potassium carbonate $\left(\mathrm{K}_{2} \mathrm{CO}_{3}\right)$, and potassium iodide $(\mathrm{KI})$ were purchased from Xilong Scientific Co., Ltd. (Shantou, China). The purified water used in the experiments was deionized water, which was prepared with a water purification system. Reinforced resin PUA was supplied by Dongguan Juncheng Chemical (Dongguan, China). HPMA, APH, SAC, MSDS, and HQ were purchased from Shanghai Adamas Reagent (Shanghai, China).

\section{Apparatus}

A 365 nm UV light source (Mightex, BLS-LCS-0365-1322) was purchased from Chengdu TaiMeng Software Co., Ltd. (Sichuan, China). A UV-vis spectrometer (Lambda 365, PerkinElmer instrument Co., Ltd.; Waltham, United States) was used to measure absorption spectra of the samples. A universal testing machine (UTM4000, San Si Zong Heng Technology Co., Ltd.; Shenzhen, China) was used to test the adhesion. The photographs were recorded using a microscope (XP-300C, Shanghai Caikon Optical Instrument Co., Ltd.; Shanghai, China).

\section{Procedures}

Synthesis of azobenzene compound M1: The synthetic process of $\mathbf{M 1}$ is shown in Figure S1. First, a solution of sodium nitrite $(3.31 \mathrm{~g})$ in water $(2 \mathrm{~mL})$ was added dropwise to a magnetically stirred solution of 4-aminophenol (4.36 g) in dilute hydrochloric acid $(2.4 \mathrm{~N}, 50 \mathrm{~mL})$ at $0{ }^{\circ} \mathrm{C}$. The mixture was stirred at $0^{\circ} \mathrm{C}$ for $30 \mathrm{~min}$ and added dropwise to a solution of o-cresol(4.32 g) in sodium hydroxide solution (20\%,15 mL) at $0{ }^{\circ} \mathrm{C}$. After complete addition, the mixture was allowed to warm to room temperature and stirred for $5 \mathrm{~h}$. After the reaction, diluted hydrochloric acid was added and the products appeared as precipitates. The mixture was acidified with dilute hydrochloric acid. The brown solid was filtered, washed with water, and dried. The crude material was purified by column chromatography on silica gel (eluent: ethyl acetate/petroleum ether $=1: 4$ ) to give 4,4'-dihydroxy3-methylazobenzene (5.02 g brown crystalline solid; yield: $55 \%)$.

${ }^{1} \mathrm{H}$ NMR (400 MHz, acetone-d6): $\delta=8.94(\mathrm{~s}, 1 \mathrm{H}), 8.87$ (s, $1 \mathrm{H}), 7.84-7.73(\mathrm{~m}, 2 \mathrm{H}), 7.69(\mathrm{~d}, J=2.4 \mathrm{~Hz}, 1 \mathrm{H}), 7.62(\mathrm{dd}$, $J=8.5,2.5 \mathrm{~Hz}, 1 \mathrm{H}), 7.04-6.92(\mathrm{~m}, 3 \mathrm{H}), 2.29(\mathrm{~s}, 3 \mathrm{H})$.

Next, a solution of 4,4'-dihydroxy-3-methylazobenzene (2 g), 6-chloro-1-hexanol (2.81 mL, 2.4 equiv), potassium carbonate ( $2.9 \mathrm{~g}, 5.0$ equiv), and potassium iodide $(0.02 \mathrm{~g}$, 0.01 equiv) in $N, N$-dimethylformamide $(40 \mathrm{~mL})$ was heated with stirring at $110{ }^{\circ} \mathrm{C}$ for $12 \mathrm{~h}$. The product was precipitated from saturated salt water, then extracted with ethyl acetate. The solvent was removed by rotary evaporation after drying with anhydrous sodium sulfate. The solid product was purified by column chromatography on silica gel (eluent:ethyl acetate/petroleum ether $=3: 2$ ) to give 4,4'-dihexanolyloxy-3-methylazobenzene (2.97 g yellow crystalline solid; yield: $79 \%$ ).

${ }^{1} \mathrm{H}$ NMR (400 MHz, chloroform- $d$ ): $\delta=7.97$ (d, $J=8.4$ $\mathrm{Hz}, 2 \mathrm{H}$ ), 7.90-7.80 ( $\mathrm{m}, 2 \mathrm{H}), 6.99$ (d, $J=8.5 \mathrm{~Hz}, 2 \mathrm{H}), 6.91$ (d, $J=8.7 \mathrm{~Hz}, 1 \mathrm{H}), 4.06(\mathrm{q}, J=6.4 \mathrm{~Hz}, 4 \mathrm{H}), 3.68(\mathrm{t}, J=6.5 \mathrm{~Hz}, 4$ H), 2.29 (s, $3 \mathrm{H}), 1.88-1.82(\mathrm{~m}, 4 \mathrm{H}), 1.66-1.43(\mathrm{~m}, 12 \mathrm{H})$.

Lastly, 4,4'-dihexanolyloxy-3-methylazobenzene (1.5 g) was dissolved in $50 \mathrm{~mL}$ of tetrahydrofuran. Triethylamine $(1.17 \mathrm{~mL})$ and acryloyl chloride $(2.7 \mathrm{~g}, 30 \mathrm{mmol})$ were added to the solution and stirred at $0{ }^{\circ} \mathrm{C}$ for $2 \mathrm{~h}$. Then the solution was filtered to remove the solvent, and the solid product was purified by column chromatography using silica gel (eluent:ethyl acetate/petroleum ether $=1: 4$ ) to give M1 (1.41 g orange crystalline solid; yield: $75 \%$ ).

${ }^{1} \mathrm{H}$ NMR (400 MHz, chloroform- $d$ ): $\delta=8.09(\mathrm{~d}, J=8.5$ $\mathrm{Hz}, 2 \mathrm{H}), 8.03-7.92$ (m, $2 \mathrm{H}), 7.03-6.98$ (m, $2 \mathrm{H}), 6.93$ (d, $J=8.7 \mathrm{~Hz}, 1 \mathrm{H}), 6.41(\mathrm{~d}, J=17.3,1.5,0.6 \mathrm{~Hz}, 2 \mathrm{H}), 6.13(\mathrm{~d}$, $J=17.2,10.4,0.7 \mathrm{~Hz}, 2 \mathrm{H}), 5.82(\mathrm{~d}, J=10.5,1.5,0.8 \mathrm{~Hz}, 2 \mathrm{H})$, 4.19 (td, $J=6.7,1.2 \mathrm{~Hz}, 4 \mathrm{H}), 4.12-4.01$ (m, $4 \mathrm{H}$ ), 2.30 (s, 3 $\mathrm{H}), 1.86(\mathrm{q}, J=16.9,6.5 \mathrm{~Hz}, 4 \mathrm{H}), 1.78-1.68(\mathrm{~m}, 4 \mathrm{H})$, $1.58-1.41$ (m, $8 \mathrm{H})$.

Sample preparation for UV-vis absorption spectroscopy test: For UV-vis absorption spectroscopy, a sandwiched cell containing the azobenzene compound M1 between two quartz plates was prepared. The sample was heated to $120^{\circ} \mathrm{C}$ and then cooled to room temperature.

Preparation of prepolymer coating for light-controlled adhesive: Azobenzene compound M1, HPMA, APH, SAC, MSDS, and HQ were dissolved in $1 \mathrm{~mL}$ of dichloromethane. After mixing, the mixture solution was coated on the substrate. The prepolymer coating was obtained after vacuum drying to remove the solvent.

UV-vis absorption spectroscopy sample preparation: A sandwiched cell containing the azobenzene compound M1 
between two cover glasses was prepared. The sample was heated to $120{ }^{\circ} \mathrm{C}$ and then cooled to room temperature.

\section{Funding Information}

S.W. thanks the National Ten Thousand Talents Program and Natural Science Foundation of Anhui Province (No. 1908085MB38); Y.L. thanks the National Natural Science Foundation of China (No. 21864010).

\section{Acknowledgment}

This work was supported by University of Science and Technology of China and Collaborative Innovation Center for Exploration of Hidden Nonferrous Metal Deposits and Development of New Materials in Guangxi.

\section{Supporting Information}

Supporting Information for this article is available online at https://doi.org/10.1055/s-0040-1721756.

\section{References}

(1) Torres, F.-G.; Troncoso, O.-P.; Cavalie, F. Mater. Sci. Eng., Proc. Conf. 2014, 34, 341.

(2) (a) Rina, M.; Kou, O.; Takuzo, A. J. Am. Chem. Soc. 2017, 139, 10072. (b) Yan, Z.; Luo, Y.; Deng, Y.; Schork, J. J. Appl. Polym. Sci. 2004, 91, 347. (c) Wang, Z.; Yu, Y.; Li, Y.; Yang, L.; Zhao, Y.; Liu, G.; Wei, Y.; Wang, X.; Tao, L. Polym. Chem. 2017, 8, 5490. (d) Wilpiszewska, K.; Czech, Z. J. Polym. Environ. 2018, 26, 1453.

(3) (a) Vinícius, G.-M.; Geovana, B.; Nei, D.; Moara, B.-S.; Rafael, B.-Z.; Marcus, S. ACS Sustainable Chem. Eng. 2017, 5, 8464. (b) Jonuzaj, S.; Cui, J.; Adjiman, C.-S. Comput. Chem. Eng. 2019, 130, 0098. (c) Kozakiewicz, J.; Kujawa-Penczek, B.; Penczek, P.; Krzysztof, P. J. Appl. Polym. Sci. 1981, 26, 3699.

(4) (a) Zhang, C.; Yu, L.; Ferdosian, F.; Vijayaraghavan, S.; Mesnager, J.; Jollet, V.; Zhao, B. Ind. Eng. Chem. Prod. Res. Dev. 2018, 57, 16318. (b) Molina-Gutiérrez, S.; Li, W.-S.-J.; Perrin, R.; Ladmiral, V.; Bongiovanni, R.; Caillol, S.; Lacroix-Desmazes, P. Biomacromolecules 2020, 21, 4514. (c) Zhou, M.; Zhai, S.; Song, T.; Zhao,
H.; Fan, Z.; Ge, F.; Zhao, Y.; Xu, Bi.; Cai, Z. J. Inorg. Organomet. Polym. Mater. 2020. Doi: 10.1007/s10904-020-01659-7.

(5) Zhao, Y.; Wu, Y.; Wang, L.; Zhang, M.; Chen, X.; Liu, M.; Fan, J.; Liu, Y.; Zhou, F.; Wang, Z. Nat. Commun. 2017, 8, 2218.

(6) Wang, C.; Li, P.; Zhang, S.; Zhang, G.; Tan, S.; Wu, Y.; Watanabe, M. Macromolecules 2020, 53, 4901.

(7) Zhou, Y.; Chen, M.; Ban, Q.; Zhang, Z.; Shuang, S.; Koynov, K.; Butt, H.-G.; Kong, J.; Wu, S. ACS Macro Lett. 2019, 8, 968.

(8) Fregoni, J.; Granucci, G.; Coccia, E.; Persico, M.; Corni, S. Nat. Commun. 2018, 9, 1.

(9) Weis, P.; Wang, D.; Wu, S. Macromolecules 2016, 49, 6368.

(10) Kravchenko, A.; Shevchenko, A.; Ovchinnikov, V.; Priimagi, A.; Kaivola, M. Adv. Mater. 2011, 23, 4174.

(11) Saydjari, A.-K.; Weis, P.; Wu, S. Adv. Energy Mater. 2017, 7, 1601622.

(12) Lv, J.-A.; Liu, Y.; Wei, J.; Chen, Y.; Qin, L.; Yu, Y. Nature 2016, 537, 179.

(13) Norikane, Y.; Hirai, Y.; Yoshida, M. Chem. Commun. 2011, 47, 1770.

(14) Akiyama, H.; Yoshida, M. Adv. Mater. 2012, 24, 2353.

(15) Xu, W.; Sun, S.; Wu, S. Angew. Chem. Int. Ed. 2019, 58, 9712.

(16) Yamauchi, M.; Yokoyama, K.; Aratani, N.; Yamada, H.; Masuo, S. Angew. Chem. Int. Ed. 2019, 58, 14173.

(17) Norikane, Y.; Uchida, E.; Tanaka, S.; Fujiwara, K.; Koyama, E.; Azumi, R.; Akiyama, H.; Kihara, H.; Yoshida, M. Org. Lett. 2014 $16,5012$.

(18) Xu, J.; Niu, B.; Guo, S.; Zhao, X.; Li, X.; Peng, J.; Deng, W.; Wu, S.; Liu, Y. Polymer 2020, 12, 901.

(19) Yue, Y.; Azumi, R.; Norikane, Y. ChemPhotoChem 2020. Doi: $10.1002 /$ cptc.202000151.

(20) Weis, P.; Tian, W.; Wu, S.; Huang, S.; Auernhammer, G.-K.; Koynov, K.; Butt, H.-G.; Wu, S. Chem. Eur. J. 2018, 24, 6494.

(21) Yue, Y.; Norikane, Y.; Azumi, R.; Koyama, E. Nat. Commun. 2018, 9, 3234.

(22) Bandara, H.-M.-D.; Burdette, S.-C. Chem. Soc. Rev. 2012, 41, 1809.

(23) Zhou, H.; Xue, C.; Weis, P.; Suzuki, Y.; Huang, S.; Koynov, K.; Auernhammer, G.-K.; Berger, R.; Butt, H.J.; Wu, S. Nat. Chem. 2017, 9, 145.

(24) Akiyama, H.; Kanazawa, S.; Okuyama, Y.; Yoshida, M.; Kihara, H.; Nagai, H.; Norikane, Y.; Azumi, R. ACS Appl. Mater. Interfaces 2014, 6, 7933.

(25) Ito, S.; Akiyama, H.; Sekizawa, R.; Mori, M.; Fukata, T.; Yoshida, M.; Kihara, H. J. Polym. Sci., Part A: Polym. Chem. 2019, 57, 806.

(26) Wu, Z.; Ji, C.; Zhao, X.; Han, Y.; Müllen, K.; Pan, K.; Yin, M. J. Am. Chem. Soc. 2019, 141, 7385.

(27) Xu, G.; Li, S.; Liu, C.; Wu, S. Chem. Asian J. 2020, 15, 547.

(28) Ito, S.; Akiyama, H.; Mori, M.; Yoshida, M.; Kihara, H. Macromol. Chem. Phys. 2019, 220, 1900105.

(29) Raftery, D.; Smyth, M.-R.; Leonard, R.-G. Int.J. Adhes. Adhes. 1997, $17,349$. 